\title{
EAl Endorsed Transactions

\section{Wireless Power Transfer Near-field Technologies for Unmanned Aerial Vehicles (UAVs): A Review}

\author{
Anh M. Le ${ }^{1,3}$, Linh H. Truong ${ }^{1,4}$, Toan V. Quyen ${ }^{1}$, Cuong V. Nguyen ${ }^{2}$, Minh T. Nguyen ${ }^{1, *}$ \\ ${ }^{1}$ Thai Nguyen University of Technology (TNUT), Viet Nam \\ ${ }^{2}$ Thai Nguyen University of Information and Communication Technology (ICTU), Viet Nam \\ ${ }^{3}$ National Chiao Tung University (NCTU), Taiwan \\ ${ }^{4}$ Tsinghua National University (NTHU), Taiwan
}

\section{Abstract}

Wireless power transfer (WPT) techniques are being popular currently with the development of midrange wireless powering and charging technology to gradually substitute the need for wired devices during charging. Unmanned Aerial Vehicles (UAVs) are also being used with many practical purposes for agriculture, surveillance, and healthcare, etc. There is a trade-off between the weight of the UAVs or their batteries and their flying time. In order to support those UAVs perform better in their tasks, WPT is applied in UAVs to recharge batteries which help to increase their working time. This paper highlights up-to-date studies that are specific to near-field WPT deploying into UAVs. The charging distances, the transfer efficiency, and transfer power, etc. are considered to provide an overview of all common problems in using and charging UAVs, especially for autonomous landing and charging. By classification and suggestions in specific problems will be provided opportunities and challenges with respect to apply near-field WPT techniques for charging the battery of UAVs and other applications in the real world.

Received on 29 November 2019; accepted on 18 January 2020; published on 31 January 2020

Keywords: Autonomous landing, capacitive coupling, inductive coupling, resonant inductive coupling, UAVs,

Copyright (C) 2020 Anh M. Le et al., licensed to EAI. This is an open access article distributed under the terms of the Creative Commons Attribution license (http://creativecommons.org/licenses/by/3.0/), which permits unlimited use, distribution and reproduction in any medium so long as the original work is properly cited.

doi:10.4108/eai.31-1-2020.162831

\section{Introduction}

The utilization of rotary-wing unmanned aerial vehicles (UAVs), with single, or multiple rotors, has gained significant interest in recent years. UAVs can be equipped with cameras, sensors, objects to deliver, etc. to be able to perform various tasks such as surveillance and military [1-3], search and rescue [4-6], remote sensing [7-9], etc. and especially will be the core of the telecommunications infrastructure supporting the Internet of Things (IoT) vision [10]. Commercial UAVs often come with lithium batteries that generate a flying time of about 20 to 40 minutes [11]. With limited operating time, UAVs may fail on several tasks, especially in missions where continuity is critical, for example in the military, monitoring production lines, etc. Based on the aforementioned problem, the

${ }^{*}$ Correspondence: Assoc. Prof. Dr. Minh T. Nguyen

nguyentuanminh@tnut.edu.vn; tuanminh.nguyen@okstate.edu
UAVs need to improve in different ways, especially in batteries or charging.

Recently, wireless power transfer (WPT) techniques that provide the power without conductive cables have evolved and provided as a promise solution in getting rid of the large grid of wires and charging single or many mobile electric devices (MEDs) at a time. Thus, MEDs can operate in harsh conditions where people can not access or the use of conductive cables to provide power is infeasible $[12,13]$. WPT supports electric power into different forms such as electric field, magnetic field, or electromagnetic radiation which is then transmitted via electric field or magnetic field to receiving devices such as mobile phones [14, $15]$, wireless sensor networks $[16,17]$ and especially for UAVs [18-21]. WPT techniques can be used to charge UAVs wirelessly, thus reduce the manpower or mechanical elements [22] to replace batteries when it is low. WPT techniques provide a promising solution and 


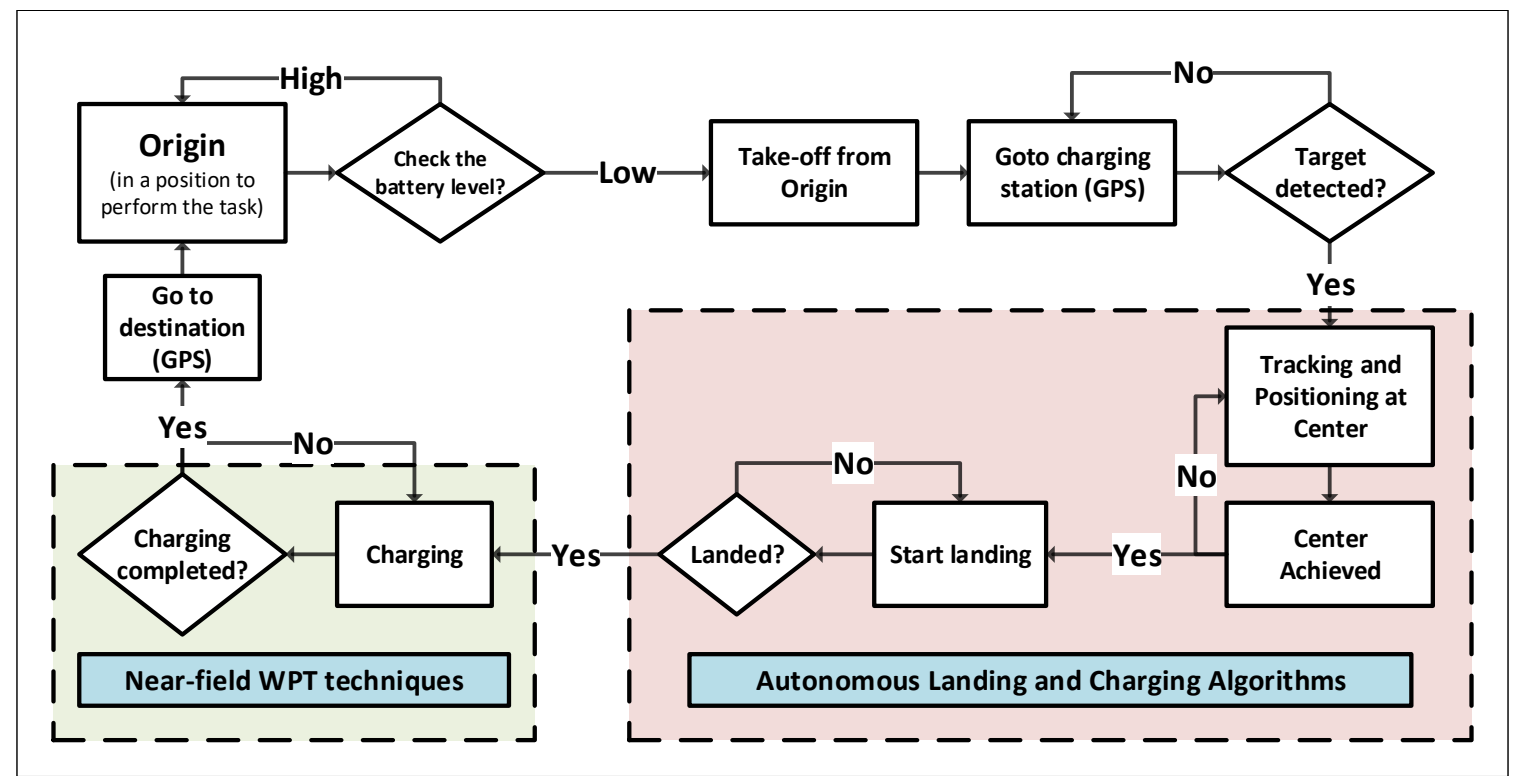

Figure 1. Autonomous landing and wireless charging algorithms for UAVs

reliable power transmission between the base-station and UAVs. These techniques help UAVS to expand its capabilities, able to complete long-term tasks that reliable and durable are required.

Wireless Power Transfer (WPT) techniques can be divided into two categories: far-field WPT (FF-WPT) and near-field WPT (NF-WPT). FF-WPT techniques have long ranges of operation, often multiple kilometer ranges. Besides, the microwave-based FF-WPT can simultaneously transmit data and energy [23, 24]. Thus, FF-WPT techniques are suitable for various applications $[25,26]$ where the power transmission efficiency is not the most important issue [27]. However, FF-WPT techniques have many drawbacks such as low power transmission efficiency $[28,29]$, safety, security, etc. that would not meet the requirement to charge UAVs.

In contrast, there are another category of WPT is near-field WPT which includes capacitive coupling (CC), inductive coupling (IC) and resonant inductive coupling (RIC). Near-field WPT techniques have the operating range is much smaller than far-field WPT, often from a few centimeters to a meter. This kind of WPT technique transfer the power based on the electric field for CC between metal electrodes or magnetic field for IC and RIC between coils of wire, respectively [30]. The advantage of near-field compared to far-field WPT techniques is that the energy transfer efficiency and the reliability of near-field WPT techniques are much higher than another one. Thus, near-field WPT techniques are more suitable for charging UAVs due to the high efficiency and potential distances ( 0 to a few meters). With the help of near-field WPT techniques,
UAVs can land on charging pads without any wire connections and perform the wireless charging process.

In this work, we focus on reviewing near-field WPT (NF-WPT) techniques for wireless charging UAVs. Besides, some recent autonomous landing and charging algorithms operated without any human intervention for persistent missions of UAVs in outdoor environments are also reviewed, as shown in Fig. 1. In Section 2, we describe the general algorithm for controlling UAVs that return from working position to the charging station along with the recently proposed methods. Near-field WPT techniques for wireless charging UAVs batteries in Section 3. Opportunities and challenges for wireless charging UAVs working in different purposes at different positions are highlighted in Section 4. Finally, in Section 5, conclusions and further works are discussed.

\section{Autonomous Landing Algorithms}

Nowadays, unmanned aerial vehicles (UAVs) are widely used in many different fields and purposes such as inspection, surveillance and policing, etc. However, one of the main reasons that limit UAVs performance is its short battery life. According to a reference [31], it takes at least half the battery life for UAVs to return to the ground charging station to charge one way. When UAVs return to the charging station, there are two main ones to charge for UAVs, namely (1) recharge locally or (2) replace batteries. Due to the inability to have the manpower to do so, both of these methods require sophisticated and precise machine components. This is very difficult when UAVs operate in difficult weather conditions such as rain or snow. In 


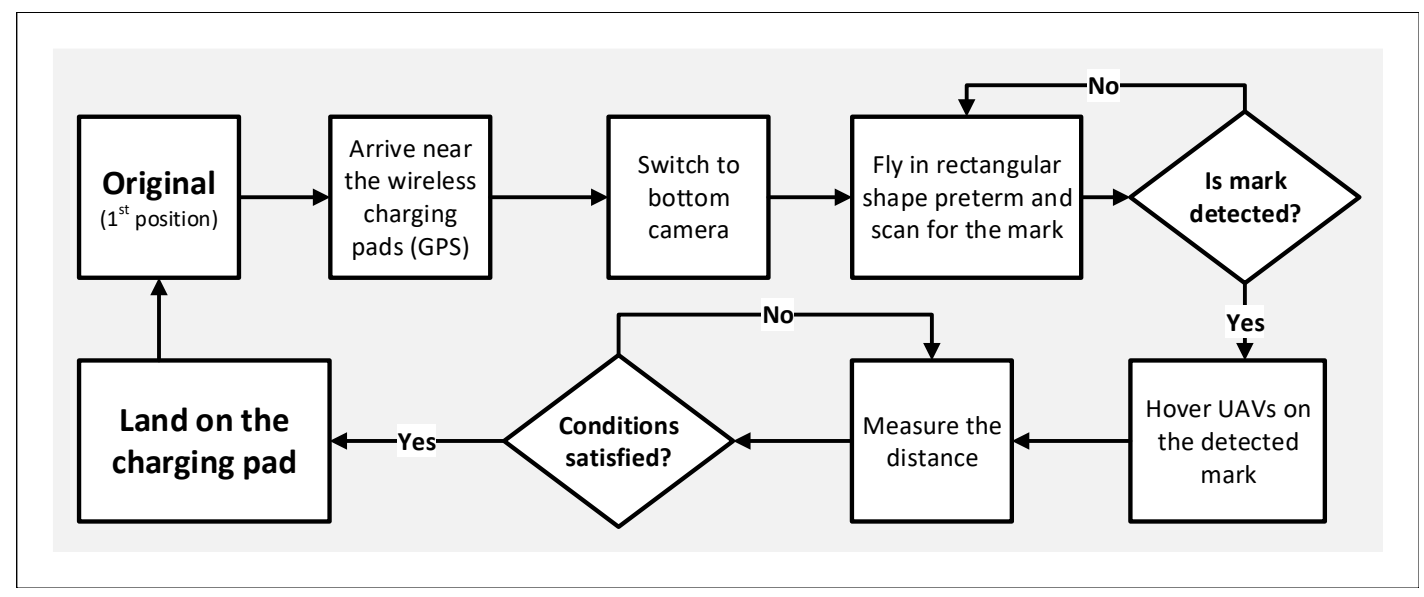

Figure 2. Automatic landing algorithm at the UAVs side

this case, autonomous landing and wireless charging via electromagnetic field are more suitable due to safer and manageable abilities for unaligned landing.

The process of returning to the charging station from the original or working position and starting charging wirelessly of UAVs consists of two main steps: (1) return from operating position and return to the charging position via GPS, (2) land on the charging pads and start charging via electric or magnetic field, as shown in Fig. 1. In order to UAVs can return to the charging station and perform the wireless charging process effectively, in addition to having to improve the efficiency of near-field charging methods, a robust and reliable algorithm also should be investigated. Thus, UAVs can land exactly on wireless charging pads and achieve the highest wireless charging performance. In this section, we review methods in both two steps as mentioned above, from the time UAVs start return to the charging area to finish the wireless charging process.

Besides the problem of controlling UAVs return to the charging station, the issue of location stability for UAVs to collect data from sensors must also be noted. However, recent UAVs stabilization algorithms have done well and depending on the different applications, the location stability requirements will vary. For example, in applications that use UAVs to monitor and send video to the server $[32,33]$, it is necessary that the video must be stable and minimize vibration. Another example is when using UAVs to collect data in the forest [34-36] about temperature, air humidity, etc. UAVs only need to stand still for a moment to collect the data from the sensors, thus it is not requiring robust and reliable position stability. For that reason, in this section, we only mention the control algorithms from when UAVs begin the return to the charging station and the process of determining the exact location of the charging point to land.
In order to perform the autonomous landing task on the charging pads, UAVs could be equipped with cameras, GPS systems, etc. and control algorithms to drive from working position to the charging station. As illustrated in Fig. 2, followed by the algorithm deployed in [31], when UAVs batteries are at a low level, it will go to the charging station by using GPS data. However, the position accuracy of the GPS system is as low as $3.0 \mathrm{~m}$ Circular Error Probable (CEP) (50\%). Thus, from $3.0 \mathrm{~m}$ of diameter, UAVs switch to bottom cameras to detect the exact location of the ground station and land on it by using marks as indicators of the charging pads. Some methods that use indicators to support UAVs landing can be mentioned as [37-40].

After UAVs detected marks, one should be built a robust and flexible control algorithms to control UAVs land exact on the charging pads. There have been many different controllers applying for UAVs such as linear controllers, nonlinear controllers, and intelligent controllers in [41]. Position and attitude control of a quad-rotor based on linear and nonlinear controllers were discussed in [42]. The linear controllers include the PID controller, Linear-quadratic regulator (LQR) controller, optimal $H_{\infty}$, and state feedback controllers. Nonlinear control techniques consist of model predictive control, back-stepping, sliding mode control, adaptive control, optimal control and Lyapunov based controllers. The other controllers use artificial intelligence tools such as neural networks, fuzzy logic inference systems, genetic algorithms, and reinforcement learning algorithms. Recently, a controller for path following of a quad-rotor UAV [43] was proposed and an attitude tracking control of a quad-rotor UAV [44] was provided. With these classical and state of art controllers, quad-rotor UAVs can be stabilized at a point in space and regulated to follow the desired path. 


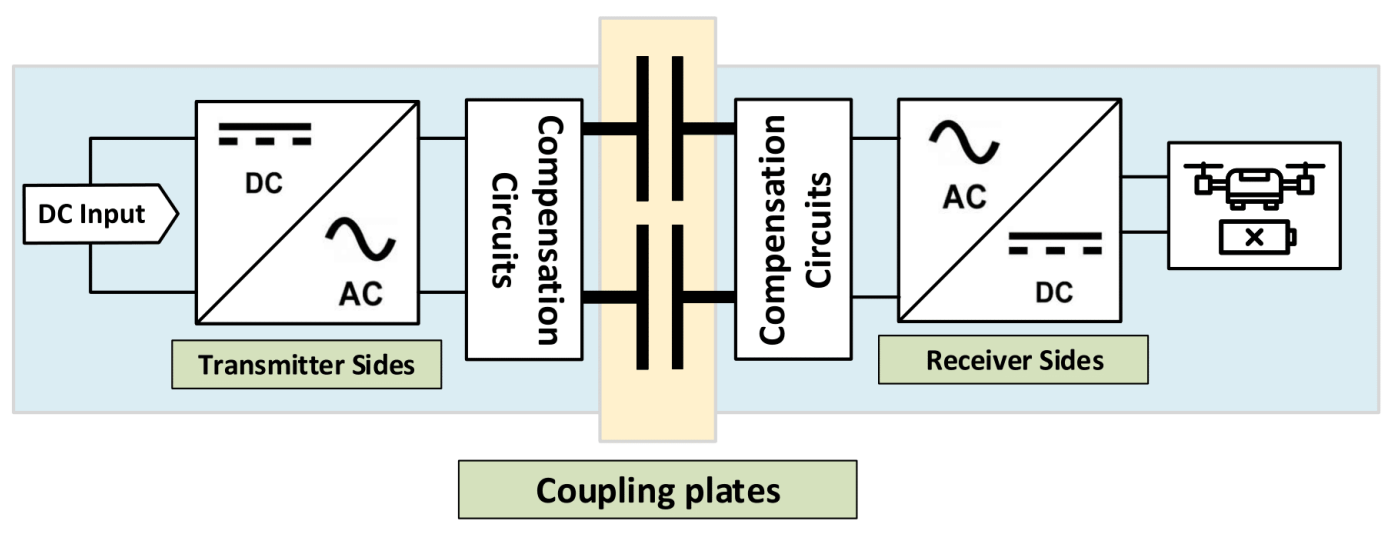

Figure 3. The structure of a typical CCWPT UAVs charging system

\section{Near-field WPT methods}

After autonomous landing on the charging pads, the system starts the process of charging wirelessly for UAVs through different methods. Once fully charged, the UAVs take-off the charging station and continue to perform the task. WPT technology is a great solution to eliminate the loss of wired transmission power generated by wire resistance. Near-field WPT techniques include capacitive coupling (CC), inductive coupling (IC) and resonant inductive coupling (RIC). In each following section, we review recent methods as well as issues and proposed methods for improvements in energy transfer performance.

\subsection{Capacitive Coupling}

In this section, we introduce the fundamental structure and operating principle of a typical capacitive coupling wireless power transfer (CCWPT). Besides, methods proposed recently to improve the efficient operation of capacitive coupling power transmission are also reviewed.

General principle. A typical capacitive coupling wireless power transfer (CCWPT) system is divided into two sides, that is, the transmitter side, Tx (left) and the receiver side, $\mathrm{Rx}$ (right), as shown in Fig. 3. On the left hand side is DC source, inverter converted DC source to $\mathrm{AC}$, compensation circuit. On the remaining side is compensation circuit, rectifier converted AC source from Tx to DC and the load, UAVs. The most important component, capacitive coupler, is placed in the middle of the system. The basic operating principle of a CCWPT system is the induced electric field between two plates via the electric field which is formed when AC source is connected and the suitable oscillated frequency.

Capacitive coupling WPT (CCWPT) techniques have several advantages that can be mentioned as low cost, high transferred power efficiency, etc. [45]. With its characteristics, CCWPT techniques are often used in application where the distance is short and low power, such as integrated circuits [46], bio-medical devices [47, 48], LED lighting [49], USB and mobile device charging $[50,51]$, especially for UAVs wireless charging [38, 52, 53]. Besides, its power can increase to $\mathrm{kW}$ level to apply for high power areas such as synchronous machine excitation [54-56], electric vehicles [57-59], etc.

CCWPT methods. We review recent methods proposed to improve the output voltage charging for UAVs via CCWPT. In addition, wireless charging systems for actual deployment UAVs are also listed. We classify proposed methods into three improved categories: (1) operating frequency; (2) compensation and (3) some deployed wireless charging system.

Operating frequency The inverter components in the CCWPT circuit have the function of converting DC from the power source to AC for wireless power transmission. Alternating current is induced and produces AC voltage on the receiver side which is formed by electrostatic induction [60]. The transmitted power is proportional to the square of the frequency, voltage, and capacitance between the plates. If the obtained capacitance is very small, the simple resonant circuit limits the transferring or high-quality factor cause the system unstable [61]. The chosen of highfrequency operation and impedance transformation is a viable solution to overcome this issue.

In order to adapt the high frequency and moderate voltage operation, Mitchell Kline et al. in [62] designed a CCWPT system using series resonances and automatic tuning loops. The proposed design ensures the circuit can operate at the pertinent frequency and achieve maximum efficiency over various values of coupling capacitance and load conditions. In addition, the choice of high frequency for wireless power transmission can be combined with the data transmission. Thus, the system can both charge and transmit data on the same 
interface. For example, in [63], Asish Koruprolu et al. proposed a system that used CCWPT techniques for both transmit power and data simultaneously over the same channel. The data transfer rate is at $170-\mathrm{kbps}$ of $1-\mathrm{MHz}$ of operating resonant frequency and the power transfer efficiency achieved $70 \%$. Alternatively, in [64], the measured power transfer efficiency, from DC input to DC output, is $90.5 \%$ and at a data rate of 119-kbps. Thus, the utilization of high frequencies in CCWPT techniques can enable UAVs to transfer data simultaneously along to the charging process. This helps UAVs save more energy due to transmit data continuously to the server.

On the other hand, Kang in [61] used higher operating frequency, $6.78-\mathrm{MHz}$ which is one of the popular ISM bands to transfer energy due to other applications instead of using $13.56 \mathrm{-MHz}$ of the operating frequency. Besides, their system consists of additional components such as a class D inverter, LC filter, and impedance transformation circuit such as step-up and step-down transformers. This addition helps improves the coupling capacitance and reduces the Q-factor. As a result, the proposed CCWPT system supplied the power to the $4-\mathrm{W} / 800-\mathrm{mA}$ output for mobile device chargers.

The aim is reducing the switching loss at the transmitted side, Yusop et al. in [65], instead of using a class-D inverter as previous authors, they used the class-E resonant inverter to perform DC-to-AC. Another main component in their design is the frequency tracking which is used to check the difference in distance between the plates so that the circuit will be adjusted in the operating frequency accordingly. The result showed that with the frequency of $1-\mathrm{MHz}$ and $0.25-\mathrm{mm}$ coupling gap, the efficiency can achieve at $96.3 \%$ and maintains within $96.3 \%$ - $91 \%$ tune the operating frequency in response to change in the coupling gap. Alternatively, Kang in [66] proposed a method to solve two issues, those are reducing the impedance of the coupling capacitor and switching loss. For the first one, the author used glass as dielectric layers that make the impedance of the capacitive coupling becomes smaller and thus transfer the power more effective. On the other hand, LLC resonant has the effect of reducing switching loss that is a turn-on and turn-off loss of the power MOSFET and rectifier, respectively. By changing some components in the circuit, the system consumes less power and at the same time higher output power.

Compensation As a typical CCWPT system illustrated in Fig. 3 in which a pair of capacitors form the coupling interface. The reactance of the capacitive coupling is inversely proportional to the operating frequency, that is, the higher the operating frequency, the smaller the reactance. If increasing this frequency to a certain threshold, the reactance of the capacitive coupling reaches the minimum value and this frequency is also called self-resonant frequency. The resonant capacitive coupling has many advantages over non-resonant capacitive coupling such as inverter can perform Zero voltage Switching or Zero Current Switching, the voltage across the capacitor and inductor are higher than that of the supply voltage, etc. However, the self-resonant frequency of a capacitive coupling system is usually very high, up to several $\mathrm{MHz}$ which will cause many problems such as switching loss, etc. In order to aid the capacitive coupling to attain resonance at a lower frequency, adding a compensation network included a combination of inductors and capacitors in series or parallel or series-parallel is a useful solution. There are various issues to consider when adding compensation networks, thus following this section are proposed methods that used compensation capacitors in both single sides and double-sides to help the system resonant at a lower frequency.

The CCWPT in [67] is an example of using the compensation network to not only reduce the resonant frequency and also increase the air-gap between the plates and still achieve energy transfer efficiency. The system is divided into two sides, the full-bridge inverter which provides $\mathrm{AC}$ and excitation to the resonant circuit in one side, compensation components which include the inductor $\mathrm{L}$, capacitor $\mathrm{C}$ and capacitive coupler in both sides and the last is the rectifier circuit that converts AC to DC in the remaining side. Due to the characteristic of basic CCWPT circuits, to achieve as high as coupler capacitance in the range of $\mathrm{pF}$, it requires a very high operating frequencies that lead to many issues. Therefore, the LC-compensation is connected with a capacitive coupler to increase the capacitance while reduce operating frequency. As a result, the system's maximum output power is around $100-\mathrm{W}$ with $150 \mathrm{~W}$ of input power at $1.5 \mathrm{MHz}$ of frequency and an air-gap of $180 \mathrm{~mm}$.

Not only focus on compensation components, in paper [68], Zhang et al. also proposed the design of improving plate structures. For plates structure, the four-plates arrange vertically instead of horizontally and different sizes to save space that very helpful in special applications such as charging for UAVs. Besides, the author used the LCL compensation components to resonate with the coupler and provide high voltage on the plates. The method achieved an efficiency of $85.87 \%$ at $1.88-\mathrm{kW}$ output power with a $150-\mathrm{mm}$ airgap distance.

The advantage of inductive power transfer (IPT) techniques is efficient power transferring at high frequency through a large air-gaps distance. Some authors in $[70,71]$ have achieved the DC-DC efficiency higher than $95 \%$ across $150-\mathrm{mm}$ air-gaps distance. However, when the frequency operation increases, the 


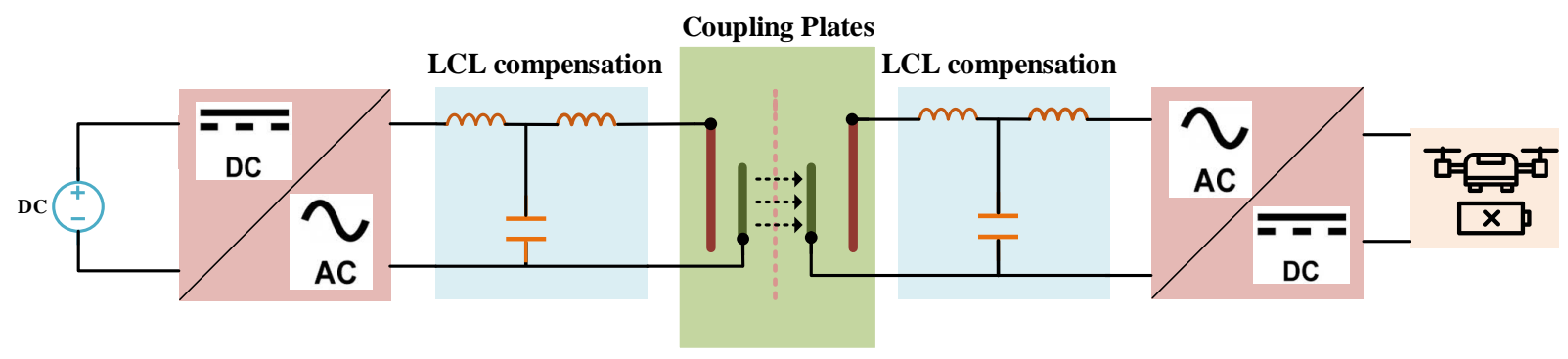

Figure 4. Double-sided LCL compensated circuit topology

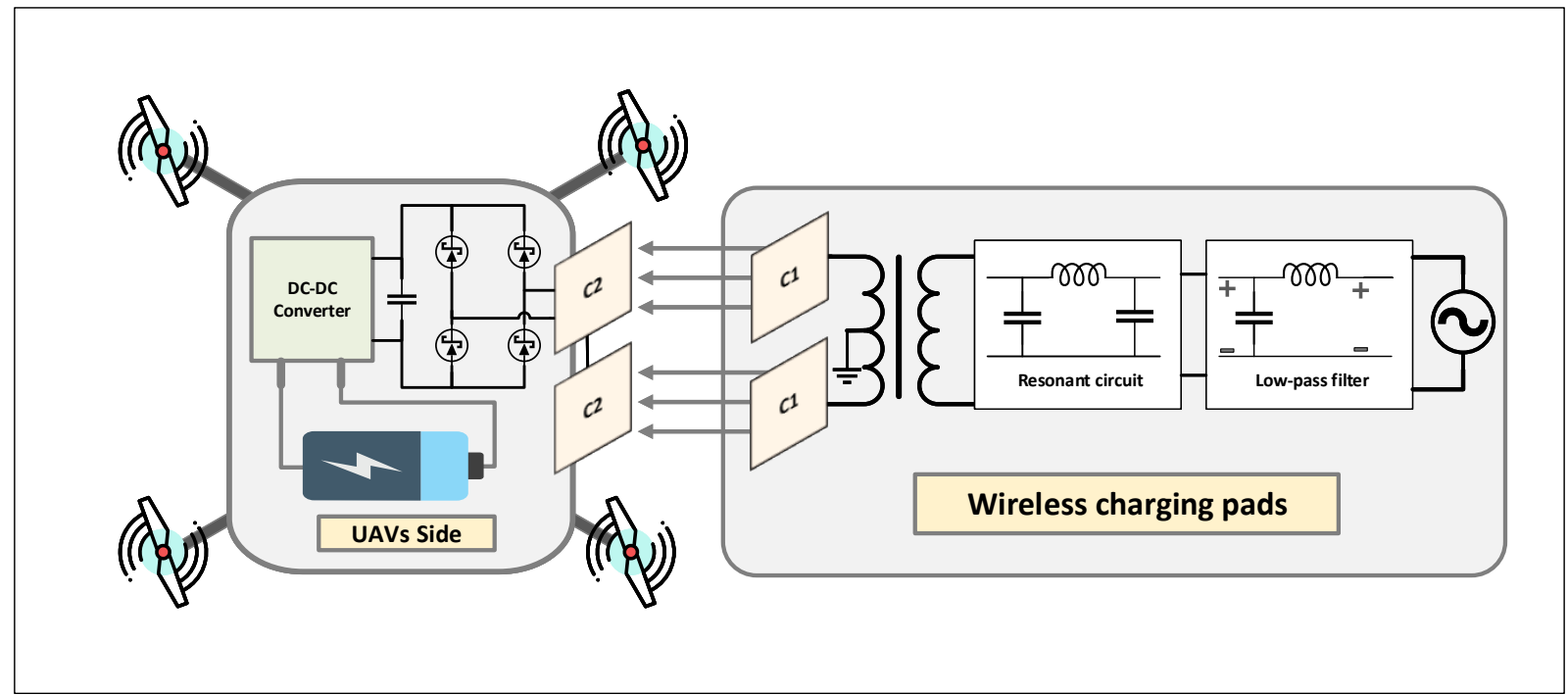

Figure 5. [69] CPT-wireless batteries charging for drone application

eddy current can cause significant temperature and can cause dangerous. In addition, to improve the inductive coupling, the IPT system usually requires ferrite plates which will increase the system cost. In the other hands, the capacitive power transfer (CPT) techniques have two main advantages: (1) the electric field does not generate eddy current, so there is no concern about temperature rising and (2) the CCWPT system used metal plates which can not only reduce the system cost and also the weight.

By combining the advantages of two approaches, Lu et al. in [72] designed a system which comprises of the coupler contained four metal structures equally divided on 2 sides, as shown in Fig. 4. Each side consists of long strips of metal sheet to increase its self-inductance. In addition, they also used LCL compensation circuit to help CCWPT system resonate with the coupler. As a result, the system achieved $73.6 \%$ efficiency of $150-W$ from DC source to DC load across an air-gap distance of $18 \mathrm{~mm}$ and $1.0 \mathrm{MHz}$ of frequency operation. The paper designed a system that can transfer the power using both magnetic and electric field simultaneously.
Similarly, Fei Lu et al. in [73] combined both inductive and capacitive coupling methods with LC-compensated topology for charging electric vehicles. The idea is both the inductive and capacitive coupling resonate together using the help of compensation components to transfer power. The output power of the system is the sum of IPT and CPT systems. With the inductive coupler size of $300 \mathrm{~mm} \times 300 \mathrm{~mm}$, the capacitve coupler size of $610 \mathrm{~mm} \times 610 \mathrm{~mm}$ and the air-gaps equal to $150 \mathrm{~mm}$, the prototype achieved $2.84-\mathrm{kW}$ power with input power of $3.0-\mathrm{kW}$. With this idea, we can improve the wireless charging efficiency for UAVs and also increase the airgap between UAVs and charging plates.

CCWPT: Deployed Systems In [53], Deepa Vincent et al. proposed a method to wireless charging for unmanned aerial vehicles (UAVs) in farming. This method increased the flight time and range of the UAV and makes it more efficient. They deployed the CCWPT system for UAVs via a master/slave arrangement where the master drone, is considered as a transmitter. The receivers or slave UAVs will have to move to the place 
of the master UAVs to be powered by the CCWPT method. This is an advantage of the proposed method since UAVs do not have to land to charge thanks to the intermediary UAVs master already connected to the base station. The paper also analyzes the method of wireless energy transfer via capacitive coupling with other applications also need to choose an appropriate air gap. In addition, to achieve the desired mutual capacitance, a high permeability dielectric is a very important element in coupling interfaces.

In [69], the author presented a design for minimum a component used in the receiver side on CCWPT for the UAVs, as shown in Fig. 5. In order to reduce the effect on the weight of the collector coil should the body of UAVs. All resonant matching circuit has been put on the side of the transmitter. The author placed on the side of the receiver a number of key components such as a rectifier circuit to obtain DC power from the transmitter coil, the DC-DC buck converter and a charging circuit to better protect the Li-Ion cells battery lifetime. The result is up to $77 \%$ efficiency with $8 \mathrm{~W}$ of transmitted power. However, it is also influenced by the rectifier components of the diode in terms of switching losses and conduction losses.

In [51], Mostafa et al. designed a circuit in which they placed components such as transformer, matching circuit and all inductors in the emitting side and the receiving sides contain only small devices using semiconductor elements for DC-DC converter and charge controlling IC for small in size and weight of UAVs. Thus, with less in size and weight, the flying time of UAVs can be longer. As the experimental result, this system can obtain $50 \%$ with the output power as $12 \mathrm{~W}$, which is enough to supply power for the 3-cell battery of drones.

\subsection{Inductive Coupling}

In recent years, the inductive coupling (IC) methods based on the resonant and magnetic principle are emerging solutions which is the performance ability of wireless power transmission with high efficiency in short distances. In this section, we review some methods to improve the IC method in the WPT system, specifically for the wireless charging technology of UAVs.

General Principle. Inductive coupling is the nearfield magnetic coupling technique of the power transfer in wireless power transmission for short-range distances [74]. The inductively coupled or magnetically coupled energy transfer uses the magnetic field, caused by an alternating current (AC) to transfer energy between coils of wire which creates an output voltage in the receiver coil. Mutual inductance (M) between two conductors is the including of an electromagnetic field (EMF) in a coil by magnetic flux lines created in another coil. It is a crucial factor to calculate the coupling coefficient $\mathrm{k}$ and the mechanism by which transformers work. The intrinsic nature of the inductive coupling techniques is similar to a transformer, included two coils: a transmitter (primary side) coil and a receiver (secondary side) coil. The coupled coils are still weak, caused the distance between a transmitter and a receiver to form an air-gap transformer, applied in electric vehicles, UAVs [75]. The distance between the two coils must be short transfer distance to each other for inductive power transfer (IPT) to work properly. With respect to the long distances, the IC technique is highly inefficient and causes energy consumption for the resistors in the primary coil.

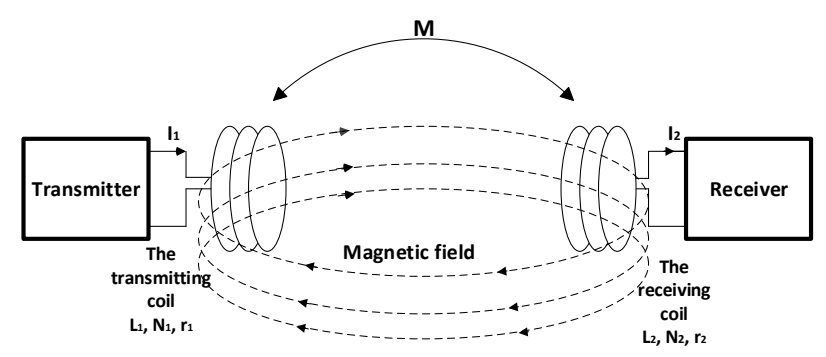

Figure 6. Typical inductive coupling system

The WPT setup has three main functional blocks consisting of a transmitter circuit, a receiver circuit, and a coupling device, as illustrated in Figure 6. Transmitting circuit converts power source as DC voltage to a high-frequency $\mathrm{AC}$ power using a power conversion circuitry. Coupling device components consist of a transmitter coil $L_{1}$ and a receiver coil $L_{2}$ as an IC device. The relationship between inductive parameters to the electric performance has become the top concern with characteristic parameters:

- A quality factor can be defined for an inductor as the ratio of the peak energy stored in the resonator in a cycle of oscillation to the energy lost per radian of the cycle. A high-quality factor indicates that the wireless system has a better response than a corresponding system having lower quality factor [74].

$$
Q=\frac{\omega L}{R}
$$

Where $\omega$ is the frequency in radian/sec.

- The coupling coefficient is the fraction of magnetic flux produced by the current in one coil.

$$
k=\frac{M}{\sqrt{L_{1} \times L_{2}}}
$$

Where $\mathrm{M}$ is mutual inductance, $L_{1}$ and $L_{2}$ are inductive of coils. 
- The turn ratio of the coil turn number is defined as the number of turn of wire in the transmitting coil to the number of turn of wire in the receiving coil. The turn ration can be expressed:

$$
\text { Turns ratio }=\frac{N_{1}}{N_{2}}
$$

- Figure of merit (FOM) is a quantity used to characterize the performance of device, system or methods relative to its alternatives.

$$
F O M=k \sqrt{Q_{t} \times Q_{r}}
$$

$Q_{t}$ and $Q_{r}$ are respectively the quality factor of transmitting and receiving coil.

- The efficiency of the WPT system is the ration of the power output to the power input, based on the coupling coefficient $(\mathrm{k})$, between the inductors and their quality (Q). It is denoted by $\eta$. The efficiency of a WPT system is given by:

$$
\eta=\frac{P_{\text {out }}}{P_{\text {in }}} \times 100
$$

Where $P_{\text {out }}$ is the output power, $P_{\text {in }}$ is the input power

Power is transferred from the transmitting side to the receiving side can be calculated in terms of the obtained efficiency of the WPT system. The maximum efficiency is for the power received and the voltage received by the receiver. This voltage can be further used to charge a given device such as a mobile phone, electric vehicles or the medical devices, etc., and in this article, it is a direct charge for UAVs. Therefore, many authors proposed methods to improve the inductive coupling method based on its principle with key parameters such as frequency, transmitted power, efficiency, and transfer distance to determine the overall system performance. These key parameters are explained in detail in the following. two directions: the internal impacts and external impacts.

Internal Impacts. The authors in [74, 76, 77] proposed to take advantage of the useful IC for both controlling remotes and power purposes. The experiment in [74] indicated that the coil shapes such as helical, spiral, rectangular and triangular effect to the shape of the inductor coil on the wireless power transmission. The MATLAB software is used to evaluate and parameterize distance, efficiency, FOM, frequency and coupling coefficient and to show the effective power transmission. The result shows that the system is achieved the efficiency is $34.2 \%$ at $\mathrm{k}=0.75$ in the range from $100 \mathrm{kHz}$ to $700 \mathrm{kHz}$. For each value $R_{L}$ $=70 \Omega, C_{\text {series }}=13-14 \mathrm{nF}$ or $C_{\text {parallel }}=6-7 \mathrm{nF}$, there are corresponding the maximum power transfer efficiency[78]. They concluded that the selection of the coupling coefficient $\mathrm{k}$ at the suitable frequency, the value of the load resistance and the value of series or parallel capacitance is very important and essential to obtain maximum efficiency.

Based on the relationship between IC parameter and electrical characteristics, Maulana et al in [76] conducted experiments by changing each value of the related key parameters, such as the distance between coupling device, transmission signal frequency, wire and coil diameter and the ratio of coil parameter in each experiment to analysis the impact of them on the power efficiency. The result illustrated that the transfer energy can reach higher efficiency of $35.85 \%$ at $40 \mu \mathrm{H}$ a low inductance value.

Yang et al analyzed inductive coupling (IC) coils in [77]. The determination of mutual inductance between wires is used in addition to the reflection load theory. Therefore, the wireless power transmission distance is extended, based on coil size requirements and the optimization of system performance. They considered in terms of the power transmission efficiency and power supply to load by calculation FOM to optimize the performance of the WPT system. The optimal distance was achieved up to 50\%, and the power transmission efficiency is more than $78 \%$ when applied to this theory for calculating the mutual inductance between coils in 2-coil and 4-coil WPT system.

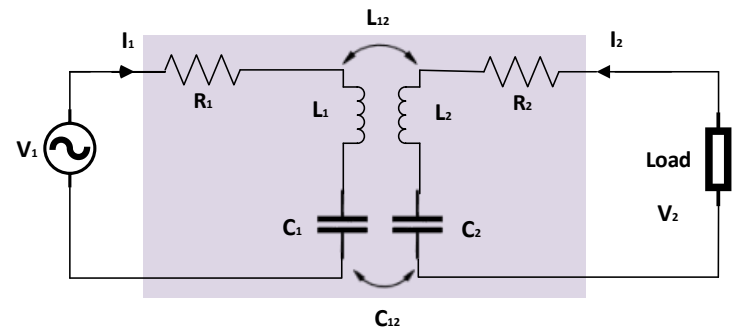

Figure 7. Equivalent network of a mixed WPT setup

A mixed WPT system with series topology determined the optimal load is proposed in [79]. The inductive wireless power transfer (IPT) and capacitive wireless power transfer (CPT) are arranged in a parallel and series topology. However, the author just did an experiment with a series topology of mixed WPT, given a system efficiency of 94,5 at $\mathrm{f}=1 \mathrm{MHz}$. To evaluate the performance of mixed WPT systems, a reference point is the maximum achievable power transfer, calculated to serve. They found that a non-zero reactance is necessary to compensate for the complex input impedance of the system. The maximum achievable power transfer was calculated to serve as a reference point for evaluating the performance of mixed WPT systems. 
Almost all aforementioned methods solved distance problems between the transmitting coil on UAVs and the receiving coil on landing area which is the big challenge of UAVs, shown in Figure 8. Besides, UAVs pose the weight challenge of its when we add more devices to UAVs.

External Impacts. The WPT technology applied to recharge the battery of the small UAVs. The problem is how to reduce the weight, size and magnetic field emission electromagnetic interference (EMI), electromagnetic compatibility (EMC) of UAVs.

With respect to the system configuration, one of the methods to reduce energy loss is the material choice as ferrite materials. They are considered as a partial magnetic core for connecting between coils. These blocks are replaced in the ambient of the transmitting coil to improve the coupling factor, whereas the receiving coil has not ferrite blocks to decrease the onboard weight $[80,81]$.

In [82], the authors proposed to optimize the WPT system from the construction of required constraints on geometry and power. The rectangular receiving coil is proved to be the best fit to support landing for UAVs. The battery of the UAVs is fully charged in about an hour therefore they used Lizt wire cable to minimize AC inductive losses. The maximum efficiency is $\eta_{\max }=93 \%$ at $N_{1}=10, N_{2}=2$, based on SeriesParallel compensation topology. The efficiency system is achieved up to $85 \%$ at all points in the charging area as $50 \times 50\left(\mathrm{~cm}^{2}\right)$. A $3 \times 2$ with $O_{w}=60 \mathrm{~mm}$ and $O_{L}=$ $70 \mathrm{~mm}$ covered this entire area [82].

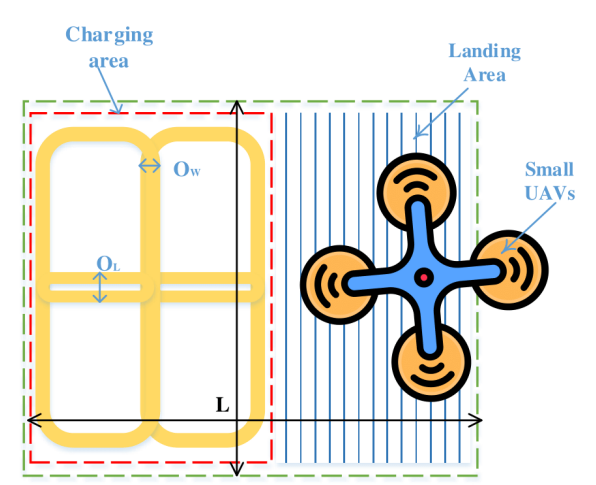

Figure 8. The WPT charging configuration

Some practical experiments [80, 83] commonly used the DJI F550 drone or small UAVs to evaluate the magnetic field levels emitted by the WPT coil current. Using a mobile positioning system for the transmitting coil to ensure a correct tolerance to misalignment conditions. The transmitting coil is placed on a 2axis system, whereas the receiving coil is placed on the landing skid of the landing gear to reduce the air gap between the coils to few millimeters and to increase the value of the coupling factor $\mathrm{k}$. UAVs start scanning the landing area from the center of the area to find the optimal point and then the input impedance is calculated moving the mechanical position system in some specific points selected by an optimized procedure to reduce the scanning time. The process ends when the measured input impedance is greater than or equal to $95 \%$ of the maximum impedance $Z_{\text {max }}$, leading to a minimum efficiency of $\eta=90 \%$ for the WPT system when using this proposed method [83].

Most recently in 2019, the principle of determining the position of the sensor is based on the measurement of the magnetic field. It varies based on the relative position of the receiver and receiver coils. The position of the receiver is located the test results of the sensor matrix and then is decided which coils among the transmission matrix to be controlled as charging coils. The authors upgraded the sensor system to find the optimal location for charging the power of the UAVs. They [84] solved the problem to improve the performance of the WPT system and to reduce electromagnetic radiation, a form of radiated or transported energy that does not require a medium in order to propagate, unlike mechanical waves such as sound and vibrations, by a novel parallel transmission coil matrix structure.

\subsection{Resonant Inductive Coupling}

Our paper in this section focuses on resonant inductive coupling (RIC) techniques of the WPT system. Firstly, the general principle is different between IC techniques and RIC techniques. Secondly, we review how to improve the efficiency of the RIC WPT system and its highlights in the application for the UAVs in the last section.

General Principle. RIC or electrodynamics induction is the near-field wireless transmission between two coils that tuned to resonate at the same frequency. The coupling devices are turned wire coils and the resonance coupled transformer. RIC is an improvement circuit of inductive coupling, operating in the midrange. Two coils at each side are added to the serial resonance capacitor, shown in Figure 9, thus adjusting the circuits to resonate at the desired frequency [75]. Greater distances can be achieved by using RIC techniques. this supports mobile applications and does not need a proper alignment between a receiver and transceiver in wireless power transmission [85].

A general configuration of the RIC-WPT system is illustrated in Figure 9, three parts: power source, transformation (resonant coupling), transmission and other parts. The transmitter and receiver coils are coupled to resonant coils via inductive coupling by mutual inductance, and the resonant coils perform 


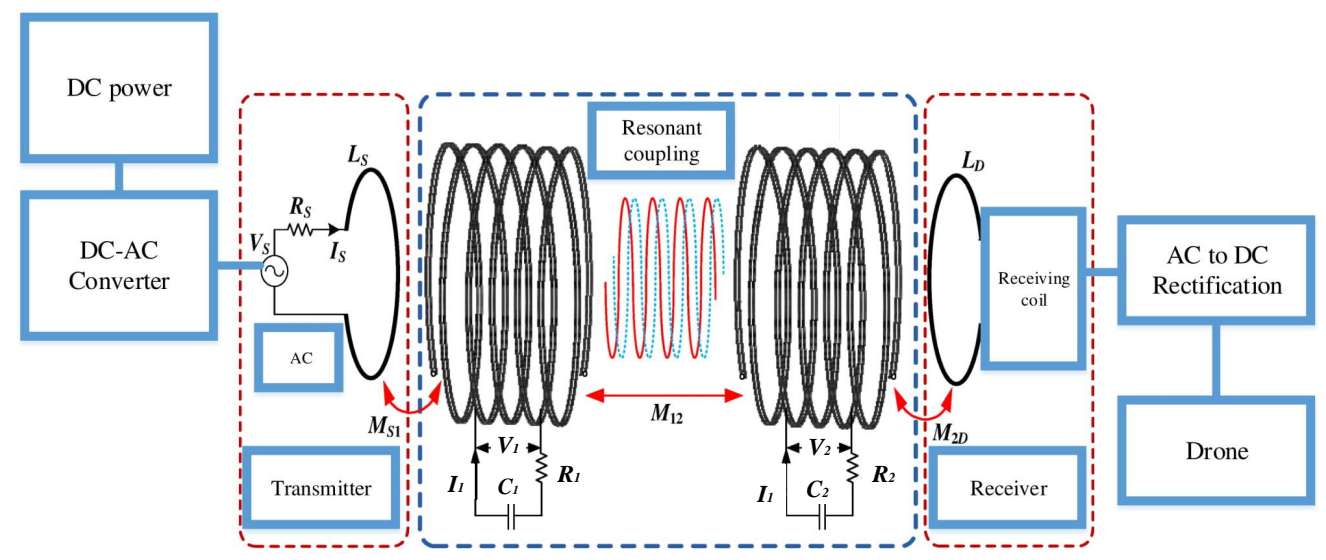

Figure 9. Typical resonant coupling system

wireless power transmission based on electromagnetic (EM) effect. The power system on the two resonators is gotten through the IC to obtain the conversion of low current/high voltage and high current/low voltage between resonators and transmitter or receiver coil. During the resonant state, the transmission loss significantly reduces due to high impedance performance. $V_{1,2}, I_{1,2}, C_{1,2}$ and $R_{1,2}$ are the voltage, current, capacitance and resistance of transmitter and receiver resonator, respectively; $I_{S, L}, L_{S, L}$, and $R_{S, L}$ are current, induction and resistance of source coil and device coil; $V_{L}$ is load voltage; $R_{L}$ is load resistance; $M_{S 1}, M_{12}$ and $M_{2 L}$ are mutual inductance between the coils [86].

The RIC technique of the WPT system has emerged to solve the problems of the efficiency in terms of the distance between the power transmission coil and the power receiver coil and the resonant frequency band. Because of a bad conducting condition, inductive coupling (IC) loses most of its energy to the air. When a distance between the transmitting and receiving coils increase, efficiency reduces significantly. The two inductor coils must have high inductance by adding resonant capacitors to increase efficiency. The maximum power transfer in the RIC technique is depended on the coupling coefficient $(\mathrm{k})$ and the quality factor $(\mathrm{Q})$ of the coil. Even if the coupling coefficient is low, power transfer can be obtained maximum by increasing Q coils. This is the most different from the IC method. But, several problems need to be overcome to implement the resonance inductive coupling method of WPT.

In the RIC-WPT system, the resonant frequency, transfer distance, coupling coefficient, and quality factor, etc. are major factors that directly affect the transfer power and transfer efficiency. There are explained in detail in the following.

Transfer power. Jose et al. [87] performed experiment with WPT using weakly coupled magnetostatic resonators. As shown in Figure 9, the author gave the condition of the coupling coefficient $\mathrm{k}$ following quality factors, given by Equation 1 and the ideal maximum efficiency $\left(\eta_{\max }\right)$ :

$$
\begin{gathered}
\kappa^{2}<<\frac{1}{1-\eta_{\max }} \cdot \frac{1}{Q_{t} Q_{r}} \\
\eta_{\max }=\frac{R_{L}}{R_{L}+R_{2}}
\end{gathered}
$$

Where $Q_{t}$ and $Q_{r}$ are respectively the quality factor of the transmitter and receiver resonators. The maximum power transfer is given by:

$$
P_{\max }=\frac{\left|V_{S}\right|^{2}}{2 R_{1}} \frac{k^{2}}{2} \frac{Q_{t} Q_{r}}{2}
$$

The experiment result is the maximum efficiency of $\eta_{\max }=95 \%$. If the distance between the two coils is larger than 4 to 8 times the coil radius $(d>4: 8 r)$, the coupling becomes weak given by Equation 6. In addition, if the quality factor is 1000 , energy transfer efficiency obtained $10 \%$ with transmission distance $\mathrm{d}$ greater than 9 times the radius $(d>9 r)$.

Samalin et al. in [88] analyzed the effect of design parameters on the transmission power of the RICWPT system. The resonant frequency band between the charging coils are coupled very strongly. Therefore, the maximum power transfer obtained is here. In addition, the impact of the vertical spacing (d) between the transmitting and receiving coils on the power transfer is revealed. For example: at the same frequency as $22.20 \mathrm{kHz}$, the power transfer efficiency is $86 \%$ with 
$\mathrm{d}=12 \mathrm{~cm}$. While power efficiency reduces to $84 \%$ at $\mathrm{d}=10 \mathrm{~cm}$.

The efficiency of WPT in the resonant inductive link is directly proportional to the magnetic field. Based on this, Sahany et al. in [89] researched the influence of the critical separation air-gap allied with the coil dimension on maximum WPT in a resonant inductive bonding. The proposed solution used the equivalent circuit model and analysis of the most accurate magnetic field to build the transmission energy to the load, then combine them with the design of coil parameters to assist in finding the optimal distance gap. Where the strongest magnetic field, it always exists an optimal separation gap. In the future, the design direction for the WPT system will be selected as the optimal separation gap between instead of changing the other design parameters.

The resonant inductive coupling includes transmitting and receiving coils. Both are connected to a compensation network (capacitors and inductors) in order to improve the transmission power efficiency of the system. The relationship between the operating frequency and the electrical loads is also indicated in [90] that the maximum power transfer occurs for a resonant frequency band and coupled region with different electrical loads. The resonant coil can create a very strong magnetic field. Therefore, the energy will be concentrated where the frequency has a proper resonant frequency. That greatly supports energy transfer performance over longer distances.

Transfer efficiency. The conventional receiver works on low efficiency. Therefore, many authors tried to find ways of getting higher efficiency. Cannon et al. in [91] designed the multiple load coils with lumped capacitors at the coil terminals and the operating frequency as 8.3 $\mathrm{MHz}$. By applying multiple receivers at the load, the resonant frequency splitting issues occur when two receivers are in close enough proximity and $Q$ resonant coupling is high which helps to increase the system efficiency. The result of the voltage drops was from $3 \mathrm{~V}$ to $0.57 \mathrm{~V}$ at the distance between two coils as $17 \mathrm{~cm}$. The transmitting power at the corresponding receiver coil is $3.3 \mathrm{~mW}$.

Kiran et al. used three-coil inductive resonant coupled for the WPT system. By calculation and simulation, then compared with the traditional structure of two coils, the efficiency of three-coils is higher because of the magnetic coupling increases. Besides, the value of the intermediate coil and external capacitors affects the output voltage and the power transfer. However, one challenge is how to choose the device size, proper capacitor and the coupling distance between the transmitter and receiver [92]. Also compared with the traditional two-coil systems, the transmission distance between the transmitting and receiving resonators can be extended up to $2 \mathrm{~m}$ by the 4 -coil systems. However, the energy efficiency system just achieved as 15\% [93]. Therefore, the impedance matching combined with the four symmetric coils (4-resonator) is used to improve the efficiency of WPT. The load impedance should match the source impedance by changing distance variations combined with adjusting the coupling parameters for achieving high efficiency. The highest efficiency reaches $92.5 \%$ at a distance of $15 \mathrm{~cm}$ and is $32.1 \%$ at a distance of $1 \mathrm{~m}$. The higher efficiency are $46.2 \%$ and $29.3 \%$ at $60 \mathrm{~cm}$ and $1 \mathrm{~m} \mathrm{[94].} \mathrm{Therefore,} \mathrm{the} \mathrm{matching} \mathrm{condition}$ is suitable for close distance.

In 2012 [95], Xue et al. presented the optimal resonant load transformation, show in Figure10. Printed spiral coils with discrete surface mount components at 13.56 $\mathrm{MHz}$ power carriers are to change and improve the factor $\mathrm{Q}$ and coupling coefficient $\mathrm{k}$. The power transfer efficiency can be achieved by $58 \%$ at $10 \mathrm{~mm}$ distance from the external coil. Kato, Masaki et al. [96] used DC-DC converter. In order to improve the transmitting efficiency, they focused on the load impedance. The load impedance is controlled by using a DC-DC converter based on the derived equation. DCDC converter changed load impedance by changing the switching duty ratio without changing load resistance. Thus, efficiency can be enhanced.

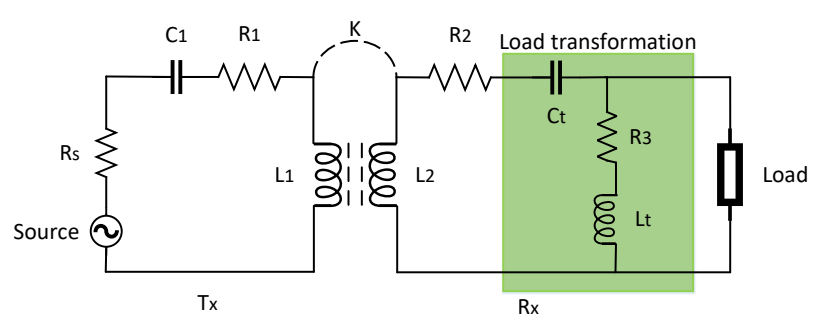

Figure 10. resonant coupling with optimal load transformation

The authors combined the RIC-WPT system with a class-E inverter as a transmission to improve the overall efficiency of the system because zero voltage switching and zero derivative switchings (ZVS/ZDS) are conditions to reduce the switching loss softswitching technique and to obtain high power-delivery efficiency. With $1 \mathrm{MHz}$ of resonant frequency and $50 \Omega$ of load resistance, the efficiency of two coils proposed system reached to $49.4 \%$ [97]. In [98], by adding more class-DE rectifier as a receiver and designing the specification for self-inductance, resistances, the system achieved a better result at $73 \%$ overall efficiency or $9.87 \mathrm{~W}(50 \Omega)$ output power, coils distance of $10 \mathrm{~cm}$.

Deploy System. A WPT system based on the resonant inductive coupling is applied to recharge the battery of small UAVs. In some experiments, the authors realized and applied to the DJI F550 drone. Besides, a Litz wire is used to reduce AC inductive losses. The 
transmitting(primary) coil is located on a terrestrial base station with some ferrite blocks to improve the coupling factor, while the receiving(secondary) coil has not ferrite blocks to reduce the on-board weight. The operation frequency is fixed to $150 \mathrm{kHz}$ [80].

In 2007, Aldhaher, and his colleagues [52] experimented to allow flying drones to be charged in mid-air. The WPT system operates in the MHz region without the need for ferrite, to do that it is integrated into softswitching resonant inverter and rectifier topologies. That helps the system operate efficiently in conditions tens of $\mathrm{MHz}$ and hundreds of watts. The mid-air WPT system is designed $\mathrm{f}=13.56 \mathrm{MHz}$ to supply power for UAVs over a distance of $12 \mathrm{~cm}$. It includes the DC/AC inverter using a load-independent class-EF inverter, transmitting coil, drone and receiving coil, rectifier and DC/DC converter using a Class-D rectifier.

Besides changing the parameters in [99] to improve the performance of the system such as $\mathrm{R}=0.265 \mathrm{~m}$, $\mathrm{C}=0.1 \mu \mathrm{F}$ for the resonant coils, $f_{\text {resonate }} \approx 189 \mathrm{kHz}$ and $\mathrm{Q} \approx 192$ (dimensionless). A $2 \Omega$ is applied for the $5 \mathrm{~V}$ supply, the system has $12.5 \mathrm{~W}$. PD controller is used to adjusting and optimizing the power transfer from a UAV. They have designed more ground sensors, to communicate the information position between the transmitter and receiver coils or between the aerial Power Transfer and static power transfer. They developed a control algorithm that is able to optimize the received power even while the aerial Power Transfer prevents it from maintaining the optimal position for power transmission. The transmitter is light and efficient enough for the UAVs to carry and operate. With static power transfer, $35 \%$ is the highest efficiency from these tests. The aerial power transfer can transfer power nearly $5 \mathrm{~W}$ continuously from the UAVs to the ground sensor.

Campi et al. [22] The RIC wireless transmission method is applied to charge UAVs during the takeoff and landing process. They are interested in the design of improved receiver and receiver coils. During the landing process, it will inevitably deviate from the position needed to charge. Therefore, an array of coils is installed on the ground, making it easier to land the charging point. They are made by a copper Litz wire to mitigate AC losses. On the other hand, the receiving coil, which is attached directly to the landing gear of the UAVs, should also be considered to reduce the weight and size of them. To complete the landing process, they also used means to communicate with the base station using a sensor circuit containing a current probe. Through practical experiments, the research has shown positive results when UAVs can automatically land and then complete charging in about an hour.

However, the existing research efforts are facing the challenges associated with UAV charging. The following section attempts to assess the opportunities and challenges of near-field WPT technologies for UAVs.

\section{Opportunities and Challenges}

Today, the world is witnessing an explosion of wireless power transfer (WPT) technology for a variety of applications such as wireless sensor nodes (WSNs), UAVs, etc. The support of this technology for UAVs' battery charging power opens up opportunities for new research direction, challenging scientists to remove the limits of technology and their applications. In this section, we take a look at the challenges and opportunities posed for the near field - WPT technology to be adopted by UAVs.

\subsection{Challenges}

From sorting and reviewing, there have been four main challenges facing energy transfer technology for UAVs. The first is the dimensions of the coils, especially the receiver coils the energy coils because they are directly placed into the body of UAVs. UAVs need to require things attached to it to have a weight limit, so as not to affect flight. In addition, if the coil size is too small, it also affects the quality factor and the coupling factor is reduced. Another fact is that there is only one receiver, but there may be more than one power transmitter. Therefore, the transmission efficiency system is affected. Yang et al. designed a new direction for the RIC method. It included multiple-input multiple-output (MIMO) in WPT systems. The multiple transmitters are used to improve the efficiency of simultaneous power transfer to multiple receivers [100]. In the process of UAVs performing the task of transmitting information data online, having a high frequency operating to transmit power for the battery of UAVs causes disturbance in the space in which UAVs transmit information in the environment, even in the long term, it also affects human health which is causing increased heat that can be a safety issue. This is the second challenge in terms of operating frequency.

For transmitting wireless energy at the near-field, the proper wireless transmission distance guarantee is an extremely important factor. The greater the transmission distance, the greater the transmission energy inevitably decreases, because the transfer distance and efficiency are inversely proportional to the transmission distance. In addition, they face the fourth challenge in terms of angular deviation in the supply power process called misalignment. One of the main limitations of inductive coupling (IC) and resonant inductive coupling (RIC) methods is sensitive to transmitting and receiving coil alignment. In order to optimize the Optimize energy transfer performance, the misalignment transmission angle between the 
Table 1. Comparison of the Near-field WPT techniques

\begin{tabular}{|c|c|c|c|}
\hline \multirow{3}{*}{$\begin{array}{c}\text { Near-field } \\
\text { Method }\end{array}$} & \multicolumn{3}{|c|}{ Non-radiative } \\
\hline & Electric Field & & tic field \\
\hline & Capacitive Coupling & Inductive Coupling & Resonant inductive coupling \\
\hline Coupling Device & Metal plate electrode & Wire coils & Tuned wire coils \\
\hline Transfer distance & Short-range & Short-range & Mid-range \\
\hline Frequency & $\mathrm{kHz}-\mathrm{MHz}$ & $\mathrm{Hz}-\mathrm{MHz}$ & $\mathrm{kHz}-\mathrm{GHz}$ \\
\hline Power & High & High & Low \\
\hline Efficiency & $85 \%$ & $85 \%$ & $40 \%$ \\
\hline Strength & Very high & Very high & High \\
\hline Multicast & No & No & Yes \\
\hline Mobility & No & No & Difficult \\
\hline Safety & Yes & Yes & Yes \\
\hline
\end{tabular}

transceiver and receiver should also be guaranteed. The longest transfer distance can be achieved only when the alignment ensures the exact requirements between the coils. In recent 2019, Li et al. in [84] designed a novel misalignment-insensitive WPT structure. The transmission coil considered as the measuring coil that adds an integrated circuit for position detection. The design helps improve transmission efficiency and at the same time effectively reduce EMR and the weight for UAVs.

\subsection{Opportunities}

The WPT technology can be completely replaced for the wire power transfer such as charge wirelessly and continuously for laptops, mobile phones, wireless sensor networks (WSNs), etc. Their invisible hands can reach out to far and far places, even reaching out to outer space. The near-field is non-radiative of WPT techniques that are much more effective for small and medium-range applications. They are relatively safe for the human body and also have no effect on the surrounding non-metallic or non-magnetic objects [101]. The development of wireless power transmission technology has led to the development of its applications. At the same time, it has had a lot of research and development to take full advantage of the technology's efficiency with each different application, especially for charging batteries for UAVs. In the future, there will be an occurrence of the methods to optimize the supply power for UAVs autonomous charging. For example, the new direction is the wireless intelligent charging device using solar energy to expand the application of UAVs $[102,103]$. The experimental results of Yang et al. shown that power transfer for UAVs is $64.87 \mathrm{~W}$ with $57.94 \%$ transfer efficiency. He also proved that the new battery charging system is less affected by angular misalignment.

The problem of energy restriction is not unique to any application such as the energy restriction provided to the sensor node system [104]. Therefore, Mittleider et al. [105] deployed a new direction for charging WSNs based on UAVs. Thanks to the magnetic resonance 
sensors, UAVs can pinpoint the exact charging position at a distance of about $15 \mathrm{~cm}$. The technique of magnetic resonance inductive of wireless energy transmission is applied to use charging UAVs for the WSN systems. The charging capacity is achieved at $5.49 \mathrm{~W}$ with a distance of about $6 \mathrm{~cm}$. Therefore, the communication range of WSNs is extended. In addition, charging for UAVs is also possible via a master UAVs. That is a lot more convenient because UAVs can be charged directly in the air. The combination of methods and applications is becoming a common trend of research. That helps them to overcome the weaknesses for each other and especially replace the human hands completely automatically.

\section{Conclusions and Future Work}

In this paper, we have briefly discussed our view on near-field techniques: capacitive coupling, inductive coupling, and resonant inductive coupling methods are addressed respectively to provide the possible ways to apply these for UAVs. This also makes clear that both inductive coupling and resonant inductive coupling are suitable choices than capacitive coupling for transmission and charging power for the battery of UAVs.

Combining all improvement methods and new designs of the above, the physical elements, circuits and the design of the resonators, etc. are main factors to perform high-transfer distance and maximum power transfer during angular or axial misalignment, to reduce power losses and the effect of EM waves when the high-oscillation frequency is used. The resonant inductive coupling is being widely used in short and mid-range wireless transmission systems like applied to extend the range of UAVs.

\section{References}

[1] S. Dean, "Local 2 investigates police secrecy behind unmanned aircraft test," Click2Houston. com, vol. 21, 2007.

[2] T. Padgett, "Drones join the war against drugs," Time Magazine, June, vol. 1, 2009.

[3] P. Lewis, "Cctv in the sky: police plan to use militarystyle spy drones," The Guardian, vol. 23, p. 1, 2010.

[4] S. G. Gupta, M. M. Ghonge, and P. Jawandhiya, "Review of unmanned aircraft system (uas)," International journal of advanced research in computer engineering $\mathcal{E}$ technology (IJARCET), vol. 2, no. 4, pp. 1646-1658, 2013.

[5] F. A. d'Oliveira, F. C. L. d. Melo, and T. C. Devezas, "High-altitude platforms-present situation and technology trends," Journal of Aerospace Technology and Management, vol. 8, no. 3, pp. 249-262, 2016.

[6] S. Chandrasekharan, K. Gomez, A. Al-Hourani, S. Kandeepan, T. Rasheed, L. Goratti, L. Reynaud, D. Grace, I. Bucaille, T. Wirth, et al., "Designing and implementing future aerial communication networks," IEEE
Communications Magazine, vol. 54, no. 5, pp. 26-34, 2016.

[7] U. Niethammer, M. James, S. Rothmund, J. Travelletti, and M. Joswig, "Uav-based remote sensing of the supersauze landslide: Evaluation and results," Engineering Geology, vol. 128, pp. 2-11, 2012.

[8] S. Herwitz, L. Johnson, S. Dunagan, R. Higgins, D. Sullivan, J. Zheng, B. Lobitz, J. Leung, B. Gallmeyer, M. Aoyagi, et al., "Imaging from an unmanned aerial vehicle: agricultural surveillance and decision support," Computers and electronics in agriculture, vol. 44, no. 1, pp. 49-61, 2004.

[9] E. R. Hunt, W. D. Hively, S. Fujikawa, D. Linden, C. S. Daughtry, and G. McCarty, "Acquisition of nir-greenblue digital photographs from unmanned aircraft for crop monitoring," Remote Sensing, vol. 2, no. 1, pp. 290305, 2010.

[10] N. H. Motlagh, T. Taleb, and O. Arouk, "Lowaltitude unmanned aerial vehicles-based internet of things services: Comprehensive survey and future perspectives," IEEE Internet of Things Journal, vol. 3, no. 6, pp. 899-922, 2016.

[11] B. Lee, S. Kwon, P. Park, and K. Kim, "Active power management system for an unmanned aerial vehicle powered by solar cells, a fuel cell, and batteries," IEEE Transactions on Aerospace and Electronic Systems, vol. 50, no. 4, pp. 3167-3177, 2014.

[12] M. T. Nguyen and T. H. Nguyen, "Wireless power transfer: A survey of techniques, and applications on communication networks," ICSES Transactions on Computer Networks and Communications (ITCNC), vol. 4, pp. 1-5, 122018.

[13] N. M. Nguyen, L. D. Nguyen, T. Q. Duong, and H. D. Tuan, "Real-time optimal resource allocation for embedded uav communication systems," IEEE Wireless Communications Letters, vol. 8, pp. 225-228, Feb 2019.

[14] M. Fareq, M. Fitra, M. Irwanto, H. S. Syafruddin, N. Gomesh, S. Farrah, and M. Rozailan, "Solar wireless power transfer using inductive coupling for mobile phone charger," in 2014 IEEE 8th International Power Engineering and Optimization Conference (PEOCO2014), pp. 473-476, IEEE, 2014.

[15] M. Fareq, M. Fitra, M. Irwanto, S. Hasan, and M. Arinal, "Low wireless power transfer using inductive coupling for mobile phone charger," in Journal of Physics: Conference Series, vol. 495, p. 012019, IOP Publishing, 2014.

[16] L. Xie, Y. Shi, Y. T. Hou, and A. Lou, "Wireless power transfer and applications to sensor networks," IEEE Wireless Communications, vol. 20, no. 4, pp. 140-145, 2013.

[17] K. Ota, H. Mizutani, R. Ishikawa, and K. Honjo, "Bi-directional wireless power transfer technology for wireless sensor/power networks," in 2013 IEEE-APS Topical Conference on Antennas and Propagation in Wireless Communications (APWC), pp. 786-789, IEEE, 2013.

[18] C. Song, H. Kim, D. H. Jung, K. Yoon, Y. Cho, S. Kong, Y. Kwack, and J. Kim, "Three-phase magnetic field design for low emi and emf automated resonant wireless power transfer charger for uav," in 2015 IEEE 
Wireless Power Transfer Conference (WPTC), pp. 1-4, IEEE, 2015.

[19] A. B. Junaid, Y. Lee, and Y. Kim, "Design and implementation of autonomous wireless charging station for rotary-wing uavs," Aerospace Science and Technology, vol. 54, pp. 253-266, 2016.

[20] M. Lu, M. Bagheri, A. P. James, and T. Phung, "Wireless charging techniques for uavs: A review, reconceptualization, and extension," IEEE Access, vol. 6, pp. 29865-29884, 2018.

[21] C. V. Nguyen, T. V. Quyen, A. M. Le, L. H. Truong, and M. T. Nguyen, "Advanced Hybrid Energy Harvesting Systems for Unmanned Ariel Vehicles (UAVs)," Advances in Science, Technology and Engineering Systems Journal, vol. 5, no. 1, pp. 34-39, 2020.

[22] T. Campi, S. Cruciani, and M. Feliziani, "Wireless power transfer technology applied to an autonomous electric uav with a small secondary coil," Energies, vol. 11, no. 2, p. 352, 2018.

[23] R. Chen, Y. Lee, and J. Sun, "Design and experiment of a loop rectenna for rfid wireless power transmission and data communication applications," Proceedings of the PIERS, Beijing, China, vol. 2327, p. 528531, 2009.

[24] A. Costanzo and D. Masotti, "Energizing 5g: Near-and far-field wireless energy and data trantransfer as an enabling technology for the $5 \mathrm{~g}$ iot," IEEE Microwave Magazine, vol. 18, no. 3, pp. 125-136, 2017.

[25] J. Ouyang, Y. Che, J. Xu, and K. Wu, "Throughput maximization for laser-powered uav wireless communication systems," in 2018 IEEE International Conference on Communications Workshops (ICC Workshops), pp. 1-6, IEEE, 2018.

[26] P. A. TP, R. Pandiarajan, and P. Raju, "Wireless power transmission to uav using laser beaming,"

[27] J. Garnica, R. A. Chinga, and J. Lin, "Wireless power transmission: From far field to near field," Proceedings of the IEEE, vol. 101, no. 6, pp. 1321-1331, 2013.

[28] H. Lyu, X. Liu, Y. Sun, Z. Jian, and A. Babakhani, "A 915-mhz far-field energy harvester with- 22-dbm sensitivity and 3-v output voltage based on antennaand-rectifier codesign," IEEE Microwave and Wireless Components Letters, vol. 29, no. 8, pp. 557-559, 2019.

[29] T. Ngo and Y.-X. Guo, "Harmonic recycling rectifier for high-efficiency far-field wireless power transfer," IEEE Transactions on Circuits and Systems II: Express Briefs, 2019.

[30] R. Erfani, F. Marefat, A. M. Sodagar, and P. Mohseni, "Transcutaneous capacitive wireless power transfer (cwpt) for biomedical implants," in 2017 IEEE International Symposium on Circuits and Systems (ISCAS), pp. 1-4, IEEE, 2017.

[31] S. Jung, T. Lee, T. Mina, and K. B. Ariyur, "Inductive or magnetic recharging for small uavs," tech. rep., SAE Technical Paper, 2012.

[32] B. Coifman, M. McCord, R. G. Mishalani, M. Iswalt, and Y. Ji, "Roadway traffic monitoring from an unmanned aerial vehicle," in IEE Proceedings-Intelligent Transport Systems, vol. 153, pp. 11-20, IET, 2006.

[33] F. Heintz, P. Rudol, and P. Doherty, "From images to traffic behavior-a uav tracking and monitoring application," in 2007 10th International Conference on Information Fusion, pp. 1-8, IEEE, 2007.

[34] D. W. Casbeer, R. W. Beard, T. W. McLain, S.-M. Li, and R. K. Mehra, "Forest fire monitoring with multiple small uavs," in Proceedings of the 2005, American Control Conference, 2005., pp. 3530-3535, IEEE, 2005.

[35] S. d'Oleire Oltmanns, I. Marzolff, K. Peter, and J. Ries, "Unmanned aerial vehicle (uav) for monitoring soil erosion in morocco," Remote Sensing, vol. 4, no. 11, pp. 3390-3416, 2012.

[36] E. Bevan, T. Wibbels, B. M. Najera, M. A. Martinez, L. A. Martinez, F. I. Martinez, J. M. Cuevas, T. Anderson, A. Bonka, M. H. Hernandez, et al., "Unmanned aerial vehicles (uavs) for monitoring sea turtles in nearshore waters," Marine Turtle Newsletter, vol. 145, no. 1, pp. 19-22, 2015.

[37] A. Junaid, A. Konoiko, Y. Zweiri, M. Sahinkaya, and L. Seneviratne, "Autonomous wireless self-charging for multi-rotor unmanned aerial vehicles," Energies, vol. 10, no. 6, p. 803, 2017.

[38] A. Raciti, S. A. Rizzo, and G. Susinni, "Drone charging stations over the buildings based on a wireless power transfer system," in 2018 IEEE/IAS 54th Industrial and Commercial Power Systems Technical Conference (IECPS), pp. 1-6, IEEE, 2018.

[39] N. Xuan-Mung and S.-K. Hong, "Improved altitude control algorithm for quadcopter unmanned aerial vehicles," Applied Sciences, vol. 9, no. 10, p. 2122, 2019.

[40] T. Campi, S. Cruciani, F. Maradei, and M. Feliziani, "Innovative design of drone landing gear used as a receiving coil in wireless charging application," Energies, vol. 12, no. 18, p. 3483, 2019.

[41] R. Amin, L. Aijun, and S. Shamshirband, "A review of quadrotor uav: control methodologies and performance evaluation," International Journal of Automation and Control, vol. 10, no. 2, pp. 87-103, 2016.

[42] G. Farid, M. Hongwei, S. M. Ali, and Q. Liwei, "A review on linear and nonlinear control techniques for position and attitude control of a quadrotor," Control and Intelligent Systems, vol. 45, no. 1, pp. 43-57, 2017.

[43] L. Qian and H. H. Liu, "Path following control of a quadrotor uav with a cable suspended payload under wind disturbances," IEEE Transactions on Industrial Electronics, 2019.

[44] H. Wang, Z. Li, H. Xiong, and X. Nian, "Robust h attitude tracking control of a quadrotor uav on so (3) via variation-based linearization and interval matrix approach," ISA transactions, vol. 87, pp. 10-16, 2019.

[45] L. Huang, A. P. Hu, A. Swain, S. Kim, and Y. Ren, "An overview of capacitively coupled power transfer-a new contactless power transfer solution," in 2013 IEEE 8th Conference on Industrial Electronics and Applications (ICIEA), pp. 461-465, IEEE, 2013.

[46] E. Culurciello and A. G. Andreou, "Capacitive interchip data and power transfer for 3-d vlsi," IEEE Transactions on Circuits and Systems II: Express Briefs, vol. 53, no. 12, pp. 1348-1352, 2006.

[47] A. M. Sodagar and P. Amiri, "Capacitive coupling for power and data telemetry to implantable biomedical microsystems," in 2009 4th International IEEE/EMBS Conference on Neural Engineering, pp. 411-414, IEEE, 
2009.

[48] R. Jegadeesan, K. Agarwal, Y.-X. Guo, S.-C. Yen, and N. V. Thakor, "Wireless power delivery to flexible subcutaneous implants using capacitive coupling," IEEE Transactions on Microwave Theory and Techniques, vol. 65, no. 1, pp. 280-292, 2016.

[49] D. Shmilovitz, A. Abramovitz, and I. Reichman, "Quasi-resonant led driver with capacitive isolation and high pf," IEEE Journal of Emerging and Selected Topics in Power Electronics, vol. 3, no. 3, pp. 633-641, 2015.

[50] K. Wang and S. Sanders, "Contactless usb-a capacitive power and bidirectional data transfer system," in 2014 IEEE Applied Power Electronics Conference and Exposition-APEC 2014, pp. 1342-1347, IEEE, 2014.

[51] T. M. Mostafa, A. Muharam, and R. Hattori, "Wireless battery charging system for drones via capacitive power transfer," in 2017 IEEE PELS Workshop on Emerging Technologies: Wireless Power Transfer (WoW), pp. 1-6, IEEE, 2017.

[52] S. Aldhaher, P. D. Mitcheson, J. M. Arteaga, G. Kkelis, and D. C. Yates, "Light-weight wireless power transfer for mid-air charging of drones," in 2017 11th European Conference on Antennas and Propagation (EUCAP), pp. 336-340, IEEE, 2017.

[53] D. Vincent, P. S. Huynh, L. Patnaik, and S. S. Williamson, "Prospects of capacitive wireless power transfer (c-wpt) for unmanned aerial vehicles," in 2018 IEEE PELS Workshop on Emerging Technologies: Wireless Power Transfer (Wow), pp. 1-5, IEEE, 2018.

[54] D. C. Ludois, J. K. Reed, and K. Hanson, "Capacitive power transfer for rotor field current in synchronous machines," IEEE Transactions on Power Electronics, vol. 27, no. 11, pp. 4638-4645, 2012.

[55] D. C. Ludois, M. J. Erickson, and J. K. Reed, "Aerodynamic fluid bearings for translational and rotating capacitors in noncontact capacitive power transfer systems," IEEE Transactions on Industry Applications, vol. 50, no. 2, pp. 1025-1033, 2013.

[56] D. C. Ludois and J. K. Reed, "Brushless mitigation of bearing currents in electric machines via capacitively coupled shunting," IEEE Transactions on Industry Applications, vol. 51, no. 5, pp. 3783-3790, 2015.

[57] J. Kim and F. Bien, "Electric field coupling technique of wireless power transfer for electric vehicles," in IEEE 2013 Tencon-Spring, pp. 267-271, IEEE, 2013.

[58] N. Sakai, D. Itokazu, Y. Suzuki, S. Sakihara, and T. Ohira, "One-kilowatt capacitive power transfer via wheels of a compact electric vehicle," in 2016 IEEE Wireless Power Transfer Conference (WPTC), pp. 1-3, IEEE, 2016.

[59] V.-B. Vu, L. B. M. Kamal, J. Tay, V. Pickert, M. Dahidah, T. Logenthiran, and V.-T. Phan, "A multi-output capacitive charger for electric vehicles," in 2017 IEEE 26th International Symposium on Industrial Electronics (ISIE), pp. 565-569, IEEE, 2017.

[60] A. Gopinath, "All about transferring power wirelessly," Electronics for You E-zine, pp. 52-56, 2013.

[61] K. H. Yi, " $6.78 \mathrm{mhz}$ capacitive coupling wireless power transfer system," Journal of Power Electronics, vol. 15, no. 4, pp. 987-993, 2015.
[62] M. Kline, I. Izyumin, B. Boser, and S. Sanders, "Capacitive power transfer for contactless charging," in 2011 Twenty-Sixth Annual IEEE Applied Power Electronics Conference and Exposition (APEC), pp. 13981404, IEEE, 2011.

[63] A. Koruprolu, S. Nag, R. Erfani, and P. Mohseni, "Capacitive wireless power and data transfer for implantable medical devices," in 2018 IEEE Biomedical Circuits and Systems Conference (BioCAS), pp. 1-4, IEEE, 2018.

[64] Y. Yao, Y. Wang, X. Liu, H. Cheng, M. Liu, and D. Xu, "Analysis, design, and implementation of a wireless power and data transmission system using capacitive coupling and double-sided lcc compensation topology," IEEE Transactions on Industry Applications, vol. 55, no. 1, pp. 541-551, 2018.

[65] Y. Yusop, S. Saat, S. K. Nguang, H. Husin, and Z. Ghani, "Design of capacitive power transfer using a class-e resonant inverter," Journal of Power Electronics, vol. 16, no. 5, pp. 1678-1688, 2016.

[66] K. H. Yi, "High frequency capacitive coupling wireless power transfer using glass dielectric layers," in 2016 IEEE Wireless Power Transfer Conference (WPTC), pp. 13, IEEE, 2016.

[67] H. Zhang, F. Lu, H. Hofmann, and C. Mi, "A loosely coupled capacitive power transfer system with lc compensation circuit topology," in 2016 IEEE Energy Conversion Congress and Exposition (ECCE), pp. 1-5, IEEE, 2016.

[68] H. Zhang, F. Lu, H. Hofmann, W. Liu, and C. C. $\mathrm{Mi}$, "A four-plate compact capacitive coupler design andlcl-compensated topology for capacitive power transfer in electric vehicle charging application," IEEE Transactions on Power Electronics, vol. 31, no. 12, pp. 8541-8551, 2016.

[69] A. Muharam, T. M. Mostafa, and R. Hattori, "Design of power receiving side in wireless charging system for uav application," in 2017 International Conference on Sustainable Energy Engineering and Application (ICSEEA), pp. 133-139, IEEE, 2017.

[70] F. Lu, H. Zhang, H. Hofmann, and C. Mi, "A high efficiency $3.3 \mathrm{kw}$ loosely-coupled wireless power transfer system without magnetic material," in 2015 IEEE Energy Conversion Congress and Exposition (ECCE), pp. 2282-2286, IEEE, 2015.

[71] S.-H. Lee and R. D. Lorenz, "Development and validation of model for $95 \%$-efficiency $220-\mathrm{w}$ wireless power transfer over a $30-\mathrm{cm}$ air gap," IEEE Transactions on Industry Applications, vol. 47, no. 6, pp. 2495-2504, 2011.

[72] F. Lu, H. Zhang, H. Hofmann, and C. C. Mi, "An inductive and capacitive integrated coupler and its lcl compensation circuit design for wireless power transfer," IEEE Transactions on Industry Applications, vol. 53, no. 5, pp. 4903-4913, 2017.

[73] F. Lu, H. Zhang, H. Hofmann, and C. C. Mi, "An inductive and capacitive combined wireless power transfer system with lc-compensated topology," IEEE Transactions on Power Electronics, vol. 31, no. 12, pp. 8471-8482, 2016. 
[74] A. Sharma and D. Kathuria, "Performance analysis of a wireless power transfer system based on inductive coupling," in 2018 International Conference on Computing, Power and Communication Technologies (GUCON), pp. 55-59, IEEE, 2018.

[75] I. Mayordomo, T. Dräger, P. Spies, J. Bernhard, and A. Pflaum, "An overview of technical challenges and advances of inductive wireless power transmission," Proceedings of the IEEE, vol. 101, no. 6, pp. 1302-1311, 2013.

[76] E. Maulana, Z. Abidin, and W. Djuriatno, "Wireless power transfer characterization based on inductive coupling method," in 2018 Electrical Power, Electronics, Communications, Controls and Informatics Seminar (EECCIS), pp. 164-168, IEEE, 2018.

[77] C.-W. Yang and C.-L. Yang, "Analysis of inductive coupling coils for extending distances of efficient wireless power transmission," in 2013 IEEE MTT-S International Microwave Workshop Series on RF and Wireless Technologies for Biomedical and Healthcare Applications (IMWS-BIO), pp. 1-3, IEEE, 2013.

[78] M. S. Habib, M. M. Rahman, A. Arshad, and S. Khan, "Analysis of power transfer efficiency of inductive coupled telemetry system for wireless power transfer," in 2014 International Conference on Computer and Communication Engineering, pp. 32-35, IEEE, 2014.

[79] B. Minnaert and N. Stevens, "Maximizing the power transfer for a mixed inductive and capacitive wireless power transfer system," in 2018 IEEE Wireless Power Transfer Conference (WPTC), pp. 1-4, IEEE, 2018.

[80] T. Campi, F. Dionisi, S. Cruciani, V. De Santis, M. Feliziani, and F. Maradei, "Magnetic field levels in drones equipped with wireless power transfer technology," in 2016 Asia-Pacific International Symposium on Electromagnetic Compatibility (APEMC), vol. 1, pp. 544547, IEEE, 2016.

[81] M. Abou Houran, X. Yang, and W. Chen, "Magnetically coupled resonance wpt: review of compensation topologies, resonator structures with misalignment, and emi diagnostics," Electronics, vol. 7, no. 11, p. 296, 2018.

[82] T. Campi, S. Cruciani, G. Rodríguez, and M. Feliziani, "Coil design of a wireless power transfer charging system for a drone," in 2016 IEEE Conference on Electromagnetic Field Computation (CEFC), pp. 1-1, IEEE, 2016.

[83] T. Campi, S. Cruciani, M. Feliziani, and F. Maradei, "High efficiency and lightweight wireless charging system for drone batteries," in 2017 AEIT International Annual Conference, pp. 1-6, IEEE, 2017.

[84] J. Li, F. Yin, L. Wang, B. Cui, and D. Yang, "Electromagnetic induction position sensor applied to anti-misalignment wireless charging for uavs," IEEE Sensors Journal, 2019.

[85] A. M. Jawad, R. Nordin, S. K. Gharghan, H. M. Jawad, and M. Ismail, "Opportunities and challenges for near-field wireless power transfer: A review," energies, vol. 10, no. 7, p. 1022, 2017.

[86] X. Zhang, H. Meng, B. Wei, S. Wang, and Q. Yang, "An improved three-coil wireless power link to increase spacing distance and power for magnetic resonant coupling system," EURASIP Journal on Wireless Communications and Networking, vol. 2018, no. 1, p. 131, 2018.

[87] J. O. Mur-Miranda, G. Fanti, Y. Feng, K. Omanakuttan, R. Ongie, A. Setjoadi, and N. Sharpe, "Wireless power transfer using weakly coupled magnetostatic resonators," Proc. IEEE ECCE, pp. 4179-4186, 2010.

[88] S. K. Samal, D. P. Kar, P. K. Sahoo, S. Bhuyan, and S. Das, "Analysis of the effect of design parameters on the power transfer efficiency of resonant inductive coupling based wireless ev charging system," in 2017 Innovations in Power and Advanced Computing Technologies (i-PACT), pp. 1-4, IEEE, 2017.

[89] S. Sahany, S. S. Biswal, P. K. Sahoo, D. P. Kar, and S. Bhuyan, "Optimal air-gap of a magnetic resonant inductive link for maximum wireless power transfer," in 2018 8th IEEE India International Conference on Power Electronics (IICPE), pp. 1-4, IEEE, 2018.

[90] D. P. Kar, S. S. Biswal, P. K. Sahoo, P. P. Nayak, and S. Bhuyan, "Selection of maximum power transfer region for resonant inductively coupled wireless charging system," AEU-International Journal of Electronics and Communications, vol. 84, pp. 84-92, 2018.

[91] B. L. Cannon, J. F. Hoburg, D. D. Stancil, and S. C. Goldstein, "Magnetic resonant coupling as a potential means for wireless power transfer to multiple small receivers," Institute of Electrical and Electronics Engineers, 2009.

[92] K. S. Kiran, M. Kumari, R. Behera, O. Ojo, and A. Iqbal, "Analysis and experimental verification of three-coil inductive resonant coupled wireless power transfer system," in 2017 National Power Electronics Conference (NPEC), pp. 84-89, IEEE, 2017.

[93] A. Kurs, A. Karalis, R. Moffatt, J. D. Joannopoulos, P. Fisher, and M. Soljačić, "Wireless power transfer via strongly coupled magnetic resonances," science, vol. 317, no. 5834, pp. 83-86, 2007.

[94] T. P. Duong and J.-W. Lee, "Experimental results of high-efficiency resonant coupling wireless power transfer using a variable coupling method," IEEE Microwave and Wireless Components Letters, vol. 21, no. 8, pp. 442-444, 2011.

[95] R.-F. Xue, K.-W. Cheng, and M. Je, "High-efficiency wireless power transfer for biomedical implants by optimal resonant load transformation," IEEE Transactions on Circuits and Systems I: Regular Papers, vol. 60 , no. 4, pp. 867-874, 2012.

[96] M. Kato, T. Imura, and Y. Hori, "Study on maximize efficiency by secondary side control using dc-dc converter in wireless power transfer via magnetic resonant coupling," World Electric Vehicle Journal, vol. 6, no. 4, pp. 858-862, 2013.

[97] S. Ansari, A. Das, and A. Bhattacharya, "Resonant inductive wireless power transfer of two-coil system with class-e resonant high frequency inverter," in 2019 6th International Conference on Signal Processing and Integrated Networks (SPIN), pp. 269-273, IEEE, 2019.

[98] T. Nagashima, X. Wei, E. Bou, E. Alarcón, and H. Sekiya, "Analytical design for resonant inductive coupling wireless power transfer system with class-e inverter and class-de rectifier," in 2015 IEEE International 
Symposium on Circuits and Systems (ISCAS), pp. 686689, IEEE, 2015.

[99] B. Griffin and C. Detweiler, "Resonant wireless power transfer to ground sensors from a uav," in 2012 IEEE International Conference on Robotics and Automation, pp. 2660-2665, IEEE, 2012.

[100] G. Yang, M. R. V. Moghadam, and R. Zhang, "Magnetic mimo signal processing and optimization for wireless power transfer," IEEE Transactions on Signal Processing, vol. 65, no. 11, pp. 2860-2874, 2017.

[101] A. Karalis, J. D. Joannopoulos, and M. Soljačić, "Efficient wireless non-radiative mid-range energy transfer," Annals of physics, vol. 323, no. 1, pp. 34-48, 2008.

[102] C. Wang and Z. Ma, "Design of wireless power transfer device for uav," in 2016 IEEE International Conference on Mechatronics and Automation, pp. 2449-2454, IEEE, 2016.

[103] C. Yang, Y. He, H. Qu, J. Wu, Z. Hou, Z. Lin, and C. Cai, "Analysis, design and implement of asymmetric coupled wireless power transfer systems for unmanned aerial vehicles," AIP Advances, vol. 9, no. 2, p. 025206 , 2019.

[104] M. T. Nguyen, H. Nguyen, A. Masaracchia, and C. Nguyen, "Stochastic-based power consumption analysis for data transmission in wireless sensor networks," EAI Endorsed Transactions on Industrial Networks and Intelligent Systems, vol. 6, no. 19, 2019.

[105] A. Mittleider, B. Griffin, and C. Detweiler, "Experimental analysis of a uav-based wireless power transfer localization system," in Experimental Robotics, pp. 357371, Springer, 2016. 